\title{
Quasistationarity in populations that are subject to large-scale mortality or emigration
}

\author{
P.K. Pollett* \\ Department of Mathematics \\ The University of Queensland
}

\begin{abstract}
We shall examine a model, first studied by Brockwell et al. [1982], which can be used to describe the long-term behaviour of populations that are subject to large-scale mortality or emigration. Populations can suffer dramatic losses when disease, such as an introduced virus, affects the population, or when food shortages occur, due to overbrowsing or significant changes in climate. However, perhaps surprisingly, such populations can survive for long periods and, although they may eventually become extinct, they can exhibit an apparently stationary regime. It is useful to be able to model this behaviour. This is particularly true of the ecological examples which motivated the present study, since, in order to properly manage these populations, it is necessary to be able to predict persistence times and to estimate the distribution of population size. We shall see that although our model predicts eventual extinction, the time till extinction can be long and the stationarity exhibited by these populations over any reasonable time scale can be explained using a quasistationary distribution.
\end{abstract}

\section{INTRODUCTION}

Our starting point is a paper by Klein [1968] (see also Scheffer [1951] and Mech [1966]) that studies populations of reindeer and moose, which, after introduction into Alaska, have suffered substantial reductions in numbers owing to overbrowsing combined with effects of severe winters; the moose population was additionally subject to Spruce Budworm infestation, and later fire. The model we shall describe, called the birth-death and catastrophe process, is particularly effective in modelling these populations (see Pakes [1987]). Another possible application of this work lies in the management of fish stocks (Holling [1973]). Although the model predicts eventual extinction, the time till extinction can be long and the surprising stationarity exhibited by the populations can be explained using a quasistationary distribution. It should be noted that the model has possible applications outside the realm of wildlife management. For example, in the market place one might wish to predict the trend in sales of a certain product, which are affected by the introduction of cheap imports or the introduction of new technology. However, in these examples, and this lies in direct contrast to the ecological examples cited above, the product may be able to adapt

\footnotetext{
*This work was funded by the Australian Research Council (Grant No. A69130032).
}

and recover quickly from catastrophic falls in sales. Nevertheless, our model might still be appropriate in instances where such adaption is less marked.

\section{THE CATASTROPHE PROCESS}

We shall use a continuous-time (time-homogeneous) Markov process $(X(t), t \geq 0)$ to model the population, where $X(t)$ is a non-negative, integervalued random variable indicating the size of the population at time $t$. We shall suppose that the process has transition rates $Q=\left(q_{j k}, j, k \geq 0\right)$ given by

$$
\begin{aligned}
q_{j, j+1} & =j \rho a, & & j \geq 0, \\
q_{j, j} & =-j \rho, & & j \geq 0, \\
q_{j, j-i} & =j \rho b_{i}, & & j \geq 2,1 \leq i<j, \\
q_{j, 0} & =j \rho \sum_{i \geq j} b_{i}, & & j \geq 1,
\end{aligned}
$$

with all other transition rates equal to 0 . Here $\rho$ and $a$ are positive, $b_{i}$ is positive for at least one value of $i$ in $\{1,2, \ldots\}$, and $a+\sum_{i \geq 1} b_{i}=1$. If $j \neq k$, $q_{j k}$ has an interpretation as the instantaneous rate at which the population size changes from $j$ to $k$. Thus, $\rho$ can be interpreted as the rate per capita at which the population size changes and, given that a change occurs, $a$ is the probability that this results in the birth of an individual and $b_{i}$ is the probability that this results in a catastrophe of size $i$ (that 
is the death or emigration of individuals). If, of the $b_{j}$ 's, only $b_{1}$ is positive, we recover the simple linear birth and death process. Clearly 0 is an absorbing state for the process and $C=\{1,2, \ldots\}$ is an irreducible class, that is, every state in $C$ can be reached by every other state in $C$. Thus if the population is initially 0 it will remain so, while if it is initially positive it will either tend to 0 or to $\infty$. It is well known and easy to prove (see for example Pakes [1987]) that the probability of extinction $\alpha_{i}$, starting with $i$ individuals, is 1 for all $i \in C$ if and only if $D$, the expected increment size, given by

$$
D=a-\sum i b_{i}=1-\sum(i+1) b_{i},
$$

is less than or equal to 0 (here and henceforth, unmarked sums shall be over $i \in C$ ). The quantity $D$ can be thought of as a drift factor and the process is said to be subcritical, critical or supercritical according as $D$ is negative, zero or positive. In the supercritical case extinction is of course still possible, and the extinction probabilities can be expressed in terms of the probability generating function $f$ of 1 minus the increment size. This is given by

$$
f(s)=a+\sum b_{i} s^{i+1}, \quad|s|<1,
$$

so that, for example, $D=1-f^{\prime}(1-)(\leq 1)$. It follows from Theorem 4 of Ezhov and Reshetnyak [1983] (see also Pakes [1987]) that when $D>0$

$$
\sum\left(1-\alpha_{i}\right) s^{i}=\frac{D s}{f(s)-s} .
$$

Thus, writing $b(s)=f(s)-s$, we see that

$$
\sum \alpha_{i} s^{i}=\frac{s}{1-s}-\frac{D s}{b(s)} \text {. }
$$

It is interesting to note that $\alpha_{i}$ tends to 0 as $i$ tends to $\infty$; roughly speaking, the larger the initial population the less likely the population is to become extinct (in the supercritical case). However, as Pakes [1987] notes, the convergence of $\alpha_{i}$ to 0 can be very slow. For example, it is easy to show, letting $s \uparrow 1$ in (1) and using L'Hôpital's rule twice, that

$$
\sum a_{i}=f^{\prime \prime}(1-) /(2 D)
$$

and that this is finite if and only if the second moment of the increment distribution is finite. Later we shall use (1) and (2), together with this condition, when evaluating quasistationary distributions.

The function $b$ we have introduced appears in connection with the theory of Markov branching processes. Indeed these processes are intimately related to the birth, death and catastrophe process, a fact which was established and exploited effectively by Pakes [1987]. We shall use one important fact from this theory: that $b(s)=0$ has a unique solution $\sigma$ on $[0,1]$. Further, $\sigma=1$ if $D \geq 0$, and $0<\sigma<1$ if $D<0$, and so $b(s) \geq 0$ on $[0, \sigma]$. Indeed, $b$ is convex on this interval.

\section{QUASISTATIONARY DISTRIBUTIONS}

We shall use two types of quasistationary distribution to describe the long-term behaviour of the process. In particular, we shall be concerned with the existence of the limits

$$
\begin{array}{r}
\lim _{t \rightarrow \infty} P(X(t)=j \mid X(0)=i, X(t)>0 \\
X(t+r)=0 \text { for some } r>0)
\end{array}
$$

and

$$
\begin{gathered}
\lim _{t \rightarrow \infty} \lim _{s \rightarrow \infty} P(X(t)=j \mid X(0)=i, X(t+s)>0 \\
X(t+s+r)=0 \text { for some } r>0)
\end{gathered}
$$

for $i, j \in C$. Thus we shall seek the limiting probability that the population size is $j$, given that extinction has not occurred, or (in the case of (4)) will not occur in the distant future, but that eventually it will; we have conditioned on eventual extinction to deal with the supercritical case, where this event has probability less than 1 .

Conditions for the existence of (3) and (4) for a general transient Markov process were obtained by Vere-Jones [1969] and Flaspohler [1974]. However, these conditions are expressed in terms of the transition probabilities of the process, which are seldom available. For conditions in terms of the transition rates, see Pollett [1986], Pollett [1988], Pollett [1989], and Parsons and Pollett [1987]. We shall attempt to describe these results in a way that is appropriate to the present context, avoiding reference to the plethora of nomenclature used in Markov chain theory. We shall start by considering the two eigenvector equations

$$
\sum m_{j} q_{j k}=-\mu m_{k}, \quad j \in C,
$$

and

$$
\sum q_{j k} x_{k}=-\mu x_{k}, \quad j \in C,
$$

where $\mu \geq 0$ and now $C$ can by any irreducible class. In the first instance we shall seek solutions $m=\left(m_{j}, j \in C\right)$ and $x=\left(x_{j}, j \in C\right)$, each with positive entries, for some $\mu \geq 0$. These are the positive left and right eigenvectors of $Q_{C}$, the transition-rate matrix restricted to $C$, corresponding to the eigenvalue $-\mu$. If $C$ is a finite set, their existence is guaranteed, at least for the eigenvalue $-\lambda$ with maximum real part (see Darroch and Seneta [1967]). If $C$ is infinite, as is the case in the present application, the situation can be considerably more complicated. For example, it might not be possible to obtain positive solutions for any value of $\mu \geq 0$. However, Vere-Jones [1969] has shown that for quasistationary distributions to exist, it is necessary that positive eigenvectors exist for some 
$\mu \geq 0$. Thus, we shall assume this to be the case in the present context. If $\lambda$ is the maximum value of $\mu$ for which positive eigenvectors exist, then we shall denote these by $m$ and $x$. Proposition 5.1 of Pollett [1988] can now be restated for our purposes as follows:

Proposition 1. If $Q$ is regular, then

(i) if $\sum m_{k} x_{k}$ converges, and either $\sum m_{k}$ converges or $\left\{x_{k}\right\}$ is bounded, then (4) exists and defines a proper probability distribution $\pi^{(2)}=\left(\pi_{j}^{(2)}, j \in C\right)$ over $C$, given by

$$
\pi_{j}^{(2)}=\frac{m_{j} x_{j}}{\sum m_{k} x_{k}}, \quad j \in C .
$$

(ii) if in addition $\sum m_{k} \alpha_{k}$ converges, then (3) exists and defines a proper probability distribution $\pi^{(1)}=\left(\pi_{j}^{(1)}, j \in C\right)$ over $C$, given by

$$
\pi_{j}^{(1)}=\frac{m_{j} \alpha_{j}}{\sum m_{k} \alpha_{k}}, \quad j \in C .
$$

Remarks. The condition that $Q$ be regular can be checked using Reuter's conditions (see Reuter [1957]). It will certainly be satisfied in the present context if we assume, as we shall, that the decrement distribution has finite mean, that is $b^{\prime}(1-)<$ 0 . The condition that $\sum m_{k} x_{k}$ converges is necessary for (7) to define a proper probability distribution over $C$. However, its real importance lies in the fact that it is sufficient for $C$ to possess the property of $\lambda$-positive recurrence (Kingman [1963]) which, for transient processes, is a notion analogous to positive recurrence for stationary processes. Indeed, in order to verify $\lambda$-positive recurrence, it suffices to show only that, for some $\mu>0$ and corresponding positive eigenvectors $m$ and $x, \sum m_{k} x_{k}<\infty$ (this follows from Theorem 5.2 of Pollett [1988]). Once $\lambda$-positive recurrence is established, Corollary 5.1 of Pollett [1988] tells us that $\lambda$, the maximal value of $\mu$, is the so-called decay parameter of $C$ (Kingman [1963]); it determines how quickly the transition probabilities $p_{j k}(t)=P(X(t)=$ $k \mid X(0)=j$ ) tend to 0 as $t$ tends to $\infty$. It then follows, from Proposition 3 of Tweedie [1974], that positive eigenvectors must exist when $\mu=\lambda$.

\section{GEOMETRIC CATASTROPHES}

Before proceeding to the general case, let us examine the important special case of when $b_{j}$ takes the form $b_{j}=b(1-q) q^{j-1}, j=1,2, \ldots$, where $b>0$, $0<q<1$ and $a+b=1$. Thus, given that a jump occurs in the size of the population, this is a birth with probability $a$ or a catastrophe with probability $b$. If a catastrophe occurs, its size has a geometric distribution. Notice that if $q=0$ we recover the linear birth and death process. We shall first solve (6), which in this case can be written as

$$
j \rho a x_{j+1}-(j \rho-\mu) x_{j}+\sum_{k=0}^{j} j \rho b_{j-k} x_{k}=0
$$

for $j \geq 1$, with the understanding that $x_{0}=0$. Substituting for $b_{j}$, multiplying by $s^{j+1}$ and then summing these equations, yields an expression for the generating function $X$, defined by $X(s)=\sum x_{k} s^{k}$, of any solution $x=\left(x_{j}, j \geq 1\right)$. The expression has a different form depending on the value of $D$, which is

$$
D=a-b /(1-q)=b(a / b-1 /(1-q))
$$

because $b(s)$ has the form

$$
\begin{aligned}
b(s) & =(1-q s)^{-1}\left\{(b+q a) s^{2}-(1+q a) s+a\right\} \\
& =a(1-s)(1-(q+b / a) s) /(1-q s) .
\end{aligned}
$$

It can be shown, although the details are somewhat tedious, that if $D=0$

$$
X(s)=\frac{s(1-q s)}{a(1-s)^{2-\theta}} \exp \left(-\frac{\mu(1-q) s}{\rho a(1-s)}\right),
$$

where $\theta=\mu q /(\rho a)$, while if $D \neq 0$

$$
X(s)=\frac{s(1-q s)}{a(1-s)^{1-\alpha}}(1-(q+b / a) s)^{\beta-1}
$$

where $\alpha=\mu /(\rho D)$ and

$$
\beta=-\frac{\alpha}{1-q}\left(\frac{b}{b+q a}\right) .
$$

The generating function is well defined for $s<\sigma$, where $\sigma$, the unique solution of $b(s)=0$ on $[0,1]$, is given by

$$
\sigma= \begin{cases}1, & \text { if } D \geq 0 \\ a /(b+q a), & \text { if } D<0\end{cases}
$$

We can now determine a candidate for the maximum value of $\mu$ for which there exists a positive right eigenvector by letting $s \uparrow \sigma$ in (10) and (11). We see that $\sum x_{k} \sigma^{k}$ converges to 0 if either $D>0$ and $\alpha>1, D<0$ and $\beta>1$, or $D=0$ and $\mu>0$. If either $D>0$ and $\alpha=1$, or $D<0$ and $\beta=1$, then the series converges to a positive number while, in the remaining cases it diverges. Thus, if $\mu$ exceeds $\lambda$, where

$$
\lambda= \begin{cases}\rho D, & \text { if } D>0 \\ 0, & \text { if } D=0 \\ -b^{-1} \rho D(1-q)(b+q a), & \text { if } D<0\end{cases}
$$

the series converges to 0 . It follows that if $x$ has non-negative entries, they must all be equal to 0 . 
However, we cannot yet deduce that $\lambda$ is maximal in the required sense. It is first necessary to show that, when $\mu=\lambda, x$ has positive entries. We shall do this by inverting the generating function $X$. After some straightforward arithmetical calculations, we find that $x$, normalized so that $x_{1}=1$, is given by

$$
x_{j}=r^{1-j}\left(\begin{array}{c}
\gamma+j-1 \\
j-1
\end{array}\right) \frac{\gamma+(1-q r)(j-1)}{\gamma+j-1}
$$

if $D>0$, where $r=a /(b+q a)$ and $\gamma=(1-$ $q r) /(1-q), x_{j}=q+(1-q) j$ if $D=0$, and,

$$
x_{j}=\left(\begin{array}{c}
\delta+j-1 \\
j-1
\end{array}\right) \frac{\delta+(1-q)(j-1)}{\delta+j-1}
$$

if $D<0$, where $\delta=1 / \gamma$. Clearly $x_{j}>0$ for each $j \geq 1$. It now remains to show that when $\mu=\lambda$ there exists a positive left eigenvector $m$ such that $\sum m_{k} x_{k}$ converges. Equations (5) take the form

$$
\begin{aligned}
(k-1) \rho a m_{k-1} & -(k \rho-\mu) m_{k} \\
& +\sum_{j \geq k+1} j \rho b_{j-k} m_{j}=0
\end{aligned}
$$

for $k \geq 1$, with the understanding that $m_{0}=0$. On substituting for $\left(b_{j}, j \geq 1\right)$, it is easy to show that the positive vector $m$ given by $m_{j}=\sigma^{j}, j \geq 1$, satisfies (12) with $\mu=\lambda$, and this is true whatever the sign of $D$. It follows that the generating function $U$, defined by $U(s)=\sum m_{k} x_{k} s^{k}$, can be evaluated in terms of the generating function for $x$ obtained on setting $\mu$ equal to $\lambda$, since clearly $U(s)=X(\sigma s)$, $s<1$. However, if $D \neq 0, \sum x_{k} \sigma^{k}$ converges, which is to say that $\sum m_{k} x_{k}$ converges. Thus, we have established, at least in the non-critical case, that $\lambda$ is maximal.

We can now use Proposition 1 to prove the existence of the quasistationary distribution $\pi^{(2)}$ when $D \neq 0$. It is easy to see that $\sum m_{k}$ converges if $D<0$ $(\sigma<1)$, while if $D>0(\sigma=1), \sum x_{k}$ converges implying that $\left\{x_{k}\right\}$ is bounded. Thus the conditions of Part (i) of the proposition are satisfied for $D \neq$ 0 and we can deduce that (4) exists and defines a proper probability distribution $\pi^{(2)}=\left(\pi_{j}^{(2)}, j \in\right.$ $C$ ), given by

$$
\pi_{j}^{(2)}=\frac{\sigma^{j} x_{j}}{\sum \sigma^{k} x_{k}}, \quad j \in C .
$$

The denominator is $X(\sigma-)$ and this can be calculated explicitly from (11) after setting $\alpha=1$ if $D>0$ and $\beta=1$ if $D<0$. On substituting into (13), we arrive at the following explicit evaluation of the quasistationary distribution $\pi^{(2)}$ :

$$
\begin{aligned}
\pi_{j}^{(2)}=(1-q)(r-1)^{1+\gamma} r^{-(\gamma+j)} & \\
& \left(\begin{array}{c}
\gamma+j-1 \\
j-1
\end{array}\right) \frac{\gamma+(1-q r)(j-1)}{\gamma+j-1}
\end{aligned}
$$

if $D>0$ and

$$
\begin{aligned}
\pi_{j}^{(2)}=(1-q r)(1-r)^{1+\delta} r^{j-1} & \\
& \left(\begin{array}{c}
\delta+j-1 \\
j-1
\end{array}\right) \frac{\delta+(1-q)(j-1)}{\delta+j-1}
\end{aligned}
$$

if $D<0$. It is interesting to note that on setting $q=0$ we obtain a result for the linear birth and death process: we see that if $a \neq b$ then (4) exists and is given by $\pi_{j}^{(2)}=(r-1)^{2} j r^{-(j+1)}$ if $r>1$, and $\pi_{j}^{(2)}=(1-r)^{2} j r^{(j-1)}$ if $r<1$, where $r=a / b$. This is consistent with the results of Pollett [1988].

We shall now prove that if $D \neq 0$, the other kind of quasistationary distribution exists as well. To do this, we shall need to check that the condition mentioned in Part (ii) of Proposition 1 is satisfied. First observe that if $D<0$, then $\alpha_{i}$, the probability of extinction starting with a population of size $i$, is equal to 1 , and so the condition is satisfied and the existence of (3) in this case is automatic. Clearly $\pi^{(1)}$ is the geometric distribution given by

$$
\pi_{j}^{(1)}=(1-\sigma) \sigma^{j-1}, \quad j \geq 1 .
$$

If $D>0$ we are required to establish that the series

$$
\sum m_{k} \alpha_{k}=\sum \alpha_{k}
$$

converges. In view of (2) it suffices to check that $f^{\prime \prime}(1-)<\infty$ (equivalently $b^{\prime \prime}(1-)<\infty$ ). On differentiating $b(s)$ twice we get $b^{\prime \prime}(1-)=$ $2 b /(1-q)^{2}$. Indeed, it is easy to invert (1). In doing so we find that $\alpha_{i}$ is proportional to $r^{-i}$. It follows that (3) exists and again defines a geometric distribution. This is given by $\pi^{(1)}=\left(\pi_{j}^{(1)}, j \geq 1\right)$, where $\pi_{j}^{(1)}=(r-1) r^{-j}$. If $q=0$ then $r=a / b$ and the specified quasistationary distributions are consistent with those found in Pollett [1988] for the linear birth and death process.

\section{THE GENERAL CASE}

Perhaps surprisingly, the process with a general catastrophe size distribution can be handled with little more difficulty. Indeed, it is possible to follow steps analogous to each of those performed above in connection with the geometric case. First, it is easy to show that the generating function $X$ of any solution to (9) can be written

$$
X(s)=\frac{s}{b(s)} \exp (-\mu B(s)), \quad s<\sigma
$$

where, for $s<\sigma, B(s)=\rho^{-1} \int_{0}^{s} d y / b(y)$. (Recall that $\sigma$ is the unique solution to $b(s)=0$ on $[0,1]$, and that $\sigma=1$ or $0<\sigma<1$ according as $D=-b^{\prime}(1-)$ is non-negative or negative.) Now, if we let $s \uparrow \sigma$ in (16), we find that $\sum x_{j} \sigma^{j}$ diverges 
if $\mu=0$, while if $\sum x_{j} \sigma^{j}$ converges it is necessary that the integral $\int_{0}^{\sigma} d y / b(y)$ diverges. Regrettably, L'Hôpital's rule is of little help to us in evaluating this sum, for the derivative of $\exp (-\mu B(s))$ is $-(\mu /(\rho b(s))) \exp (-\mu B(s))$. However, we can say that if $\sum x_{j} \sigma^{j}$ converges to a positive number, it is necessary that $\mu=\lambda$, where $\lambda=-\rho b^{\prime}(\sigma-)$ $(\geq 0)$. Let us now consider the left eigenvector equation (12). If one attempts a solution of the form $m_{j}=t^{j}$, where $t>0$, then the $k=1$ equation implies that $\mu=-\rho b^{\prime}(t-)$ and the other equations imply that $b(t)=0$. Since we require $\mu \geq 0$ it is necessary that $b^{\prime}(t-) \leq 0$. Thus, for $m$ to be of this form it is necessary that $t=\sigma$ and $\mu=\lambda$. We have shown that when $\mu=\lambda$, (12) admits the positive solution $m=\left(m_{j}, j \geq 1\right)$, given by $m_{j}=\sigma^{j}$, no matter what the form of $\left(b_{j}, j \geq 1\right)$. It follows that the convergence or otherwise of $\sum m_{k} x_{k}$ depends, as before, on the behaviour of $X$ near $s=\sigma$. If we can establish that this series converges and that $\lambda$ is maximal in the required sense, then, by Proposition 1, (4) exists and defines a quasistationary distribution $\pi^{(2)}=\left(\pi_{j}^{(2)}, j \geq 1\right)$ given by (13). Further, the existence of (3) will then be automatic, at least when $D>0$, since then $\alpha_{i}=1$ for all $i \geq 1$ and $0<\sigma<1$; the quasistationary distribution $\pi^{(1)}=\left(\pi_{j}^{(1)}, j \geq 1\right)$ will be given by (14) (a result of Pakes [1987]). If $D>0$ then, on consideration of (2) and (15), it is clear that if $f^{\prime \prime}(1-)<\infty$ (equivalently $b^{\prime \prime}(1-)<\infty$ ), then (3) will exist and $\pi^{(1)}$ will be given by $\pi_{j}^{(1)}=\alpha_{j} / \sum \alpha_{k}$. It follows that $\pi^{(1)}$ will have a probability generating function given by (1).

All of this rests on establishing that the series $\sum x_{j} \sigma^{j}$ converges and that $\lambda$ is the maximal value of $\mu$ for which positive eigenvectors exist. It seems difficult to obtain necessary conditions for the convergence of this series. However, in view of Theorem 6.2 of Pakes [1987], it is clear that a sufficient condition for $\sum x_{j} \sigma^{j}$ to converge is that either $D<0$, or, $D>0$ and $b$ can be written as $b(s)=D(1-s)+(1-s)^{2} L\left((1-s)^{-1}\right)$, where $L$ is a slowly varying function. Note that $b$ has this form in the case of "geometric catastrophes" because it can be shown that

$$
L(x)=\frac{b x}{(1-q)((1-q) x+q)},
$$

and so clearly $L(x t) \sim L(x)$ for large $t$. If one of these conditions can be satisfied, then it is possible to deduce that $\lambda$ is maximal using an argument based on Theorem 5.1 of Pollett [1988]. Thus, we have been able to establish the existence of the quasistationary distribution (4) in the subcritical case, and in the supercritical case when a regularity condition on $b$ is satisfied. In the subcritical case, (3) also exists under these conditions, while in the su- percritical case an extra condition, that the decrement distribution has finite second moment, is required.

\section{DISCUSSION}

To illustrate our results, we shall consider the effect on the mean population size (under the quasistationary distributions) of varying the parameters of the model. For simplicity, we shall restrict our attention to the case where the size of catastrophes has a geometric distribution. Let $\phi=a / b$ and let $c=1 /(1-q)$, so that $\phi$ represents the ratio of births to catastrophes, while $c(\geq 1)$ is the average size of a catastrophe. Observe that the process is subcritical, critical or supercritical according as $c$ is greater than $\phi$, equal to $\phi$, or less than $\phi$, and that if $\phi \leq 1$ the process cannot be supercritical. Expressions for the mean of the two quasistationary distributions can be obtained either directly, or by differentiating the appropriate generating functions. Let $m^{(1)}$ and $m^{(2)}$ be, respectively, the mean of $\pi^{(1)}$ and the mean of $\pi^{(2)}$. Then, it is easy to show that $m^{(1)}=-\phi+\phi^{2} /(\phi-c)$ if $1 \leq c<\phi, m^{(1)}=1+\phi+\phi^{2} /(c-\phi)$ if $c>\phi$, $m^{(2)}=1-2 \phi-2 c+2 \phi^{2} /(\phi-c)$ if $1 \leq c<\phi$, and $m^{(2)}=1+2 \phi((1+\phi) c-\phi) /(c(c-\phi))$ if $c>\phi$. Recall that in the critical case no quasistationary distribution exists; the limits in (3) and (4) are identically 0 for all $i$ and $j$.

Let us first determine the effect of varying $\phi$ with $c$ fixed. Figure 1 illustrates the results for the linear birth and death process obtained on setting $c=1$. Notice that $m^{(1)}$ underestimates $m^{(2)}$ uniformly.

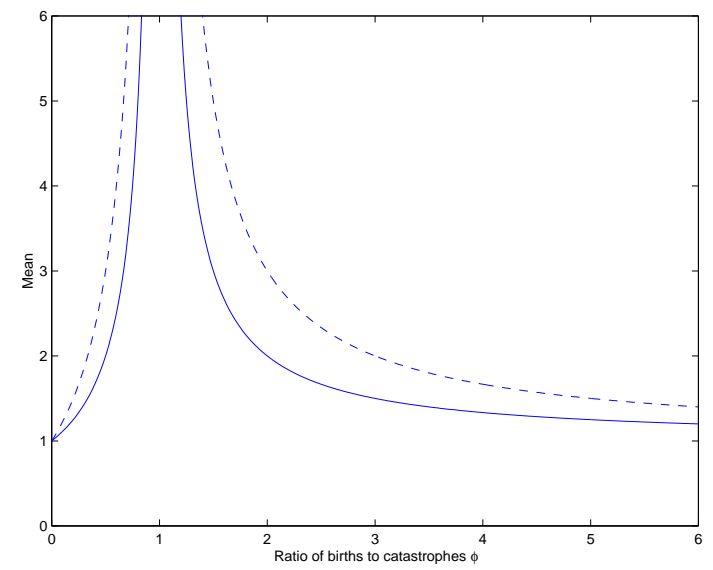

Figure 1: Quasistationary distribution means $m^{(1)}$ (solid) and $m^{(2)}$ (dashed) for $c=1$.

This is indicative of the fact that, in general, (3) assigns more mass closer to 0 than does (4) and, further, it is consistent with the fact that (4) is more appropriate for describing the behaviour of the population early in its evolution, well before extinction occurs. Notice also that both means are large for 
near-critical values of $\phi$, in which case genuine quasistationary behaviour obtains. If $\phi$ is large, the likelihood of extinction is rather small yet, surprisingly, both distributions assign all their mass near 1 .

Next we shall observe the effect of varying the mean catastrophe size $c$ when $\phi$ is fixed. In order to allow for the possibility of a supercritical case, we shall choose a value of $\phi$ greater than 1 , say $\phi=2$. Figure 2 illustrates the results. The trends are similar to

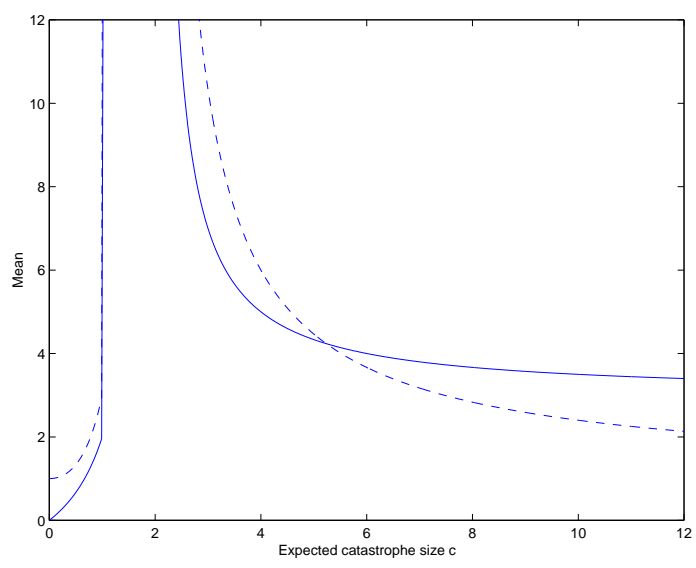

Figure 1: Quasistationary distribution means $m^{(1)}$ (solid) and $m^{(2)}$ (dashed) for $\phi=1$.

those already observed, in particular, for the supercritical and near-critical cases. However, notice that in the subcritical case the graphs cross and, for sufficiently large values of $c, m^{(2)}$ underestimates $m^{(1)}$. Further, the limiting values as $c$ becomes large are not the same, the difference between these values being $\phi$. That both distributions assign nearly all their mass near 1 , is consistent with the fact that the time till extinction is rather short.

\section{REFERENCES}

Brockwell, P., Gani, J. and S. Resnick, Birth, immigration and catastrophe processes, Adv. Appl. Probab., 14, 709-731, 1982.

Darroch, J. and E. Seneta, On quasi-stationary distributions in absorbing continuous-time finite Markov chains, J. Appl. Probab., 4, 192-196, 1967.

Ezhov, I. and V. Reshetnyak, A modification of the branching process, Ukranian Math. J., 35, 2833, 1983.

Flaspohler, D., Quasi-stationary distributions for absorbing continuous-time denumerable Markov chains, Ann. Inst. Statist. Math., 26, 351356, 1974.

Holling, C., Resilience and stability of ecological systems, Ann. Rev. Ecol. Systematics, 4, 1-23, 1973.
Kingman, J., The exponential decay of Markov transition probabilities, Proc. London Math. Soc., 13, 337-358, 1963.

Klein, D., The introduction, increase, and crash of reindeer on St. Matthew Island, J. Wildlife Man., 32, 351-367, 1968.

Mech, L., The wolves of Ilse Royale, Fauna of the National Parks: U.S. Fauna Series, 7, 1966.

Pakes, A., Limit theorems for the population size of a birth and death process allowing catastrophes, J. Math. Biol., 25, 307-325, 1987.

Parsons, R. and P. Pollett, Quasistationary distributions for some autocatalytic reactions, $J$. Statist. Phys., 46, 249-254, 1987.

Pollett, P., On the equivalence of $\mu$-invariant measures for the minimal process and its $q$-matrix, Stoch. Process. Appl., 22, 203-221, 1986.

Pollett, P., Reversibility, invariance and $\mu$-invariance, Adv. Appl. Probab., 20, 600-621, 1988.

Pollett, P., The generalized Kolmogorov criterion, Stoch. Process. Appl., 33, 29-44, 1989.

Reuter, G., Denumerable Markov processes and the associated contraction semigroups on $l$, Acta Math., 97, 1-46, 1957.

Scheffer, V., The rise and fall of a reindeer herd, Sci. Monthly, 73, 356-362, 1951.

Tweedie, R., Some ergodic properties of the feller minimal process, Quart. J. Math. Oxford, 25, 485-495, 1974.

Vere-Jones, D., Some limit theorems for evanescent processes, Austral. J. Statist., 11, 67-78, 1969. 